PROCEEDINGS OF THE

AMERICAN MATHEMATICAL SOCIETY

Volume 140, Number 2, February 2012, Pages 377-391

S 0002-9939(2011)10904-3

Article electronically published on May 31, 2011

\title{
A GENERALIZATION OF MAX NOETHER'S THEOREM
}

\author{
RENATO VIDAL MARTINS
}

(Communicated by Bernd Ulrich)

\begin{abstract}
Max Noether's theorem asserts that if $\omega$ is the dualizing sheaf of a nonsingular nonhyperelliptic projective curve, then the natural morphisms $\operatorname{Sym}^{n} H^{0}(\omega) \rightarrow H^{0}\left(\omega^{n}\right)$ are surjective for all $n \geq 1$. This is true for Gorenstein nonhyperelliptic curves as well. We prove that this remains true for nearly Gorenstein curves and for all integral nonhyperelliptic curves whose non-Gorenstein points are unibranch. The results are independent and have different proofs. The first one is extrinsic, the second intrinsic.
\end{abstract}

\section{INTRODUCTION}

Let $C$ be an integral and complete curve of arithmetic genus $g$ over an algebraically closed field $k$. Let $\omega$ be its dualizing sheaf. We start by introducing Max Noether's celebrated theorem exactly as it is in [1, p. 117]: if $C$ is nonsingular and nonhyperelliptic, then the homomorphisms

$$
\operatorname{Sym}^{n} H^{0}(C, \omega) \longrightarrow H^{0}\left(C, \omega^{n}\right)
$$

are surjective for $n \geq 1$.

The same reference says that the result is a consequence of projective normality of extremal curves, i.e., curves whose genus matches Castelnuovo's bound. Indeed, extremal curves are always projectively normal and this is a general fact proved in [1. pp. 113-117] for nonsingular curves, but the reader should note that, since the Riemann-Roch theorem and Clifford's theorem have versions for singular curves (44, App.], [7, pp. 186-191], [9, p. 108]), the same proof holds for all integral curves as well.

Now assume $C$ is Gorenstein. Then $\omega$ defines a morphism $\kappa: C \rightarrow \mathbb{P}^{g-1}$. Let $C^{\prime}:=\kappa(C)$ be the canonical model of $C$. M. Rosenlicht proved in [7 that $C^{\prime}$ is extremal and that $\kappa$ is an isomorphism if $C$ is nonhyperelliptic. Therefore Max Noether's Theorem actually holds for all Gorenstein nonhyperelliptic curves.

We start this article with Theorem 2.7, which extends Rosenlicht's result almost in the same way as above to a different class of curves, called nearly Gorenstein in [5]. They have just one non-Gorenstein point for which the local ring is almost Gorenstein, a desirable (though restrictive) property introduced by R. Fröberg and V. Barucci in 2]. The first difference between our approach and Rosenlicht's is that we derive projective normality from linear normality. Most of the work was already done in [5, Lem. 5.4.(1)], which we rephrase and re-prove in Lemma 2.2 .

Received by the editors September 14, 2009 and, in revised form, November 16, 2010.

2010 Mathematics Subject Classification. Primary 14H20; Secondary 14H45, 14H51.

Key words and phrases. Singular curve, non-Gorenstein curve, Max Noether theorem.

(C)2011 American Mathematical Society 377

Reverts to public domain 28 years from publication 
The second difference is that we deal with $\widehat{C}$, the blowup along $\omega$, instead of with $C$.

In order to deal with this problem intrinsically, we were motivated by a strong result due to M. Rosenlicht [7, Thm. 17]. It asserts that if $C$ is nonhyperelliptic, then the birational map between $C$ and the canonical model $C^{\prime}$ [cf. Definition[2.9] is regular on $C^{\prime}$. His proof, especially in the non-Gorenstein case, is focused on the local rings of these sorts of points and the stalks of the dualizing sheaf at them; the technique was the computation of values of differentials. Indeed, in the last two pages of the article, where the core of Rosenlicht's proof lies, there is only one paragraph where he does not compute values. So with this same tool we prove the following statement, which is our main result: if $C$ is a nonhyperelliptic curve whose non-Gorenstein points are unibranch, then Max Noether's assertion holds (Theorem 3.7).

The hypothesis assuming that the non-Gorenstein points are unibranch is due to property (8) below, which describes the possible values of differentials that are regular at a given point of $C$. In the general case, it is not easy to deal with this property [9, Thm.2.11], as Rosenlicht did, within our context. In fact, we point out that Max Noether's assertion implies Rosenlicht's [cf. Remark 2.10]. In other words, our task is a little harder. This leads us to think that the multibranch problem deserves more work. In this one, the reader should note that although in Section 3 we always assume that the non-Gorenstein points are unibranch, it is just Step 2 of the proof of Lemma 3.2 that must be extended in order to get a sharper result. The question as to whether Noether's statement holds for arbitrary integral curves remains open.

\section{Generalization By means of An extrinsic argument}

Let $C$ be an integral and complete curve of arithmetic genus $g$ over an algebraically closed field $k$ of arbitrary characteristic. Let $\mathcal{O}:=\mathcal{O}_{C}$ be the structure sheaf on $C$. According to [1, p. 140] we make the following definition.

Definition 2.1. A curve $C \subset \mathbb{P}^{r}$ is $n$-normal if the hypersurfaces of degree $n$ cut out the complete linear series $\left|\mathcal{O}_{C}(n)\right|$. A 1-normal curve is said to be linearly normal and a curve is said to be projectively normal if it is $n$-normal for all $n \geq 1$.

We just note that neither linearly normal nor projectively normal implies that the curve is normal. For instance, the first two examples of singular curves, i.e., the plane nodal and cuspidal curves, are projectively normal though not normal schemes. Moreover, any integral nonhyperelliptic Gorenstein singular curve of genus $g$ can be embedded as a projectively normal curve in $\mathbb{P}^{g-1}$, as implicitly pointed out in the Introduction.

Lemma 2.2. Let $C$ be a nondegenerate curve of degree $d$ in $\mathbb{P}^{r}$. If $d<2 r$ or if $d=2 r$ and $h^{1}\left(\mathcal{O}_{C}(1)\right)>0$, then $C$ is linearly normal iff it is projectively normal.

Proof. Sufficiency is immediate. To prove necessity, consider the left exact sequence

$$
0 \longrightarrow H^{0}\left(\mathcal{O}_{C}(n-1)\right) \stackrel{u}{\longrightarrow} H^{0}\left(\mathcal{O}_{C}(n)\right) \stackrel{v}{\longrightarrow} H^{0}\left(\mathcal{O}_{H \cap C}(n)\right),
$$

where $H$ is the ambient hyperplane.

Let $V_{n}$ denote the image of $H^{0}\left(\mathcal{O}_{\mathbb{P}^{r}}(n)\right)$ in $H^{0}\left(\mathcal{O}_{C}(n)\right)$, and set $W_{n}:=v\left(V_{n}\right)$. Then $\operatorname{dim}\left(W_{n}\right) \geq \min \{d, n(r-1)+1\}$ by [1, Lem., p. 115]. Hence

$$
h^{0}\left(\mathcal{O}_{C}(n)\right)-h^{0}\left(\mathcal{O}_{C}(n-1)\right) \geq \min \{d, n(r-1)+1\} .
$$


Also, if equality holds in (2) , then $v\left(H^{0}\left(\mathcal{O}_{C}(n)\right)\right)=W_{n}$ since both sides have the same dimension. So $H^{0}\left(\mathcal{O}_{C}(n)\right)$ is spanned by $V_{n}$ and $\operatorname{Im}(u)$. But $u\left(V_{n-1}\right) \subset V_{n}$. So if $H^{0}\left(\mathcal{O}_{C}(n-1)\right)=V_{n-1}$, then $\operatorname{Im}(u) \subset V_{n}$ and hence $H^{0}\left(\mathcal{O}_{C}(n)\right)=V_{n}$. So, for each $n \geq 2$, we conclude that the equality in (2) along with $H^{0}\left(\mathcal{O}_{C}(n-1)\right)=V_{n-1}$ implies that $H^{0}\left(\mathcal{O}_{C}(n)\right)=V_{n}$.

Therefore, assuming $C$ is linearly normal, i.e., $V_{1}=H^{0}\left(\mathcal{O}_{C}(1)\right)$, if we prove that equality holds in (2) for $n \geq 2$, then, by induction, $H^{0}\left(\mathcal{O}_{C}(n)\right)=V_{n}$ for every $n \geq 1$; that is, $C$ is projectively normal.

In order to prove so, set $h(n):=h^{1}\left(\mathcal{O}_{C}(n)\right)$. From the long exact sequence

$$
H^{0}\left(\mathcal{O}_{C}(n-1)\right) \rightarrow H^{0}\left(\mathcal{O}_{C}(n)\right) \rightarrow H^{0}\left(\mathcal{O}_{H \cap C}(n)\right) \rightarrow H^{1}\left(\mathcal{O}_{C}(n-1)\right) \rightarrow H^{1}\left(\mathcal{O}_{C}(n)\right)
$$

it is easily checked that the bound (2) is equivalent to the following bound:

$$
d-(h(n-1)-h(n)) \geq \min \{d, n(r-1)+1\} .
$$

Here $h(n-1)-h(n) \geq 0$ because $H^{1}\left(\mathcal{O}_{H \cap C}(n)\right)=0$.

Write $d=2 r-a$ for $a \geq 0$. We have

$$
(n(r-1)+1)-d=(n-2)(r-1)-1+a .
$$

If $r=1$, then $C=\mathbb{P}^{1}$, which is projectively normal. So assume $r \geq 2$. For $n \geq 2$, the right side is nonnegative unless $a=0$ and $n=2$.

Hence, for $n \geq 3$, the right side of (3) is equal to $d$. But $h(n-1)-h(n) \geq 0$. Therefore, equality holds in (3), and $h(n-1)=h(n)$. But, by Serre's Theorem, $h(n)=0$ for $n \gg 0$. So $h(n)=0$ for $n \geq 2$.

Suppose $a>0$. Then similarly, equality holds in (3) for $n=2$ too, and $h(1)=0$. So equality holds in (2) for $n \geq 2$, as desired.

Finally, instead, suppose $a=0$. Then $d=2 r$ and $h(1)>0$ by hypothesis. Now, take $n=2$ in (3), getting $2 r-h(1)$ on the left as $h(2)=0$, and $2 r-1$ on the right. Hence, $h(1)=1$ and equality holds in (3) for $n=2$. So equality holds in (2) for $n \geq 2$, as desired. The proof is now complete.

A curve $C$ is said to be hyperelliptic if there is a morphism $C \rightarrow \mathbb{P}^{1}$ of degree 2. Let $\omega_{C}$, or simply $\omega$, denote the dualizing sheaf. A point $P \in C$ is said to be Gorenstein if $\omega_{P}$ is a free $\mathcal{O}_{P}$-module. A curve $C$ is said to be Gorenstein if all of its points are Gorenstein or, equivalently, if $\omega$ is invertible. For instance, every nonsingular curve is Gorenstein, and also every hyperelliptic (possibly singular) curve is Gorenstein as well [5, Prop. 2.6.(2)].

Given any integral scheme $A$, any map $\alpha: A \rightarrow C$ and a sheaf $\mathcal{G}$ on $C$, set

$$
\mathcal{O}_{A} \mathcal{G}:=\alpha^{*} \mathcal{G} / \operatorname{Torsion}\left(\alpha^{*} \mathcal{G}\right)
$$

Let $\nu: \bar{C} \rightarrow C$ be the normalization map. Set $\overline{\mathcal{O}}:=\nu_{*}\left(\mathcal{O}_{\bar{C}}\right)$. We denote by $\mathcal{C}$ the conductor of $\overline{\mathcal{O}}$ into $\mathcal{O}$. We also set $\bar{\omega}:=\nu_{*}\left(\omega_{\bar{C}}\right)$ and $\overline{\mathcal{O}} \omega:=\nu_{*}\left(\mathcal{O}_{\bar{C}} \omega\right)$.

Given any coherent sheaf $\mathcal{F}$ on $C$, set

$$
\mathcal{F}^{n}:=\operatorname{Sym}^{n} \mathcal{F} / \operatorname{Torsion}\left(\operatorname{Sym}^{n} \mathcal{F}\right) .
$$

If $\mathcal{F}$ is invertible, then clearly $\mathcal{F}^{n}=\mathcal{F}^{\otimes n}$. Let $\phi: \operatorname{Sym}^{n} H^{0}(\mathcal{F}) \rightarrow H^{0}\left(\mathcal{F}^{n}\right)$ be the natural morphism. We set

$$
H^{0}(\mathcal{F})^{n}:=\phi\left(\operatorname{Sym}^{n} H^{0}(\mathcal{F})\right)
$$

and just warn the reader not to confuse it with $H^{0}(\mathcal{F})^{\oplus n}$, which is different. 
Call $\widehat{C}:=\operatorname{Proj}\left(\oplus \omega^{n}\right)$ the blowup of $C$ along $\omega$. Let $\beta: \widehat{C} \rightarrow C$ be the natural morphism. Set $\widehat{\mathcal{O}}=\beta_{*}\left(\mathcal{O}_{\widehat{C}}\right)$ and $\widehat{\mathcal{O}} \omega:=\beta_{*}\left(\mathcal{O}_{\widehat{C}} \omega\right)$.

Definition 2.3. Let $k(C)$ be the function field of $C$. For $E \subset k(C)$ let $E^{n}$ be the set of all finite sums of products of $n$ elements from $E$. Let $\Omega_{k(C) \mid k}$ be the set of differentials. For any $z \in \Omega_{k(C) \mid k}$, set

$$
\begin{aligned}
W_{z} & :=\left\{f \in k(C) \mid f z \in H^{0}(\omega)\right\}, \\
V_{z, P} & :=\left\{f \in k(C) \mid f z \in \omega_{P}\right\}, \\
W_{z, n} & :=\bigcap_{P \in C} V_{z, P}^{n} .
\end{aligned}
$$

If $\varphi: \widetilde{C} \rightarrow C$ is a morphism and $\widetilde{\omega}:=\varphi_{*}\left(\omega_{\widetilde{C}}\right)$, set

$$
\begin{aligned}
\widetilde{W}_{z} & :=\left\{f \in k(C) \mid f z \in H^{0}(\widetilde{\omega})\right\}, \\
\widetilde{V}_{z, P} & :=\left\{f \in k(C) \mid f z \in \widetilde{\omega}_{P}\right\}, \\
\widetilde{W}_{z, n} & :=\bigcap_{P \in C} \widetilde{V}_{z, P}^{n} .
\end{aligned}
$$

Definition 2.4. We fix, throughout this paper, a differential $x \in H^{0}(\omega)$ such that $(\overline{\mathcal{O}} \omega)_{P}=\overline{\mathcal{O}}_{P} x$ for every singular point $P \in C$. Such a differential exists because $H^{0}(\omega)$ generates $\overline{\mathcal{O}} \omega$, as proved in [7, p. 188 top], the singular points of $C$ are of finite number and $k$ is infinite since it is algebraically closed.

Example 2.5. Take $C$ to be the projective closure of Spec $k\left[t^{3}, t^{4}, t^{5}\right]$. It has just one singular point, say $P$, which corresponds to $t=0$ if $C$ is given parametrically; we also have $\mathcal{O}_{P}=k\left[t^{3}, t^{4}, t^{5}\right]_{\left(t^{3}, t^{4}, t^{5}\right)}$, where the notation refers to localization. The normalization is $\bar{C}=\mathbb{P}^{1}$, taken as the projective closure of Spec $k[t]$ and so $k(C)=k(\bar{C})=k(t)$. There is only one point $\bar{P} \in \bar{C}$ over $P$, and we have $\overline{\mathcal{O}}_{P}=\mathcal{O}_{\bar{P}}=k[t]_{(t)}$. So the genus of $C$ is $g=\bar{g}+\operatorname{dim}\left(\overline{\mathcal{O}}_{P} / \mathcal{O}_{P}\right)=0+2=2$, where $\bar{g}$ is the genus of $\bar{C}$. We claim that $x=d t / t^{3}$ has the desired property of the above definition. First, according to [8, p. $191 \mathrm{mid}]$, we can use the residue formula to compute $\omega_{P}$; we have that $V_{x, P}$ is the largest among the $\mathcal{O}_{P}$-fractional ideals $F$ in $k(C)$ such that $\operatorname{Res}_{\bar{P}}(f x)=0$ for every $f \in F$. One can check that $V_{x, P}=\mathcal{O}_{P}+t \mathcal{O}_{P}$ as we note, for instance, that $t^{2} \notin V_{x, P}$ since $\operatorname{Res}_{\bar{P}}\left(t^{2} x\right)=\operatorname{Res}_{\bar{P}}(d t / t)=1$. If $t^{2} \notin V_{x, P}$, the same holds for all $t^{-n}$ for $n \geq 1$ since $V_{x, P}$ is an $\mathcal{O}_{P}$-fractional ideal and $t^{n} \in \mathcal{O}_{P}$ for all $n \geq 3$. Therefore $\omega_{P}=\left(\mathcal{O}_{P}+t \mathcal{O}_{P}\right) x$, which is not a free $\mathcal{O}_{P}$-module, so $P$ is non-Gorenstein. Now $(\overline{\mathcal{O}} \omega)_{P}=\left(\overline{\mathcal{O}}_{P}\left(\mathcal{O}_{P}+t \mathcal{O}_{P}\right)\right) x=\overline{\mathcal{O}}_{P} x$ and the claim follows. Note, however, that $(\overline{\mathcal{O}} \omega)_{P}$ is not a free $\mathcal{O}_{P}$-module, but $\left(\mathcal{O}_{\bar{C}} \omega\right)_{\bar{P}}=\mathcal{O}_{\bar{P}} \omega_{P}=\mathcal{O}_{\bar{P}} x$, so $\left(\mathcal{O}_{\bar{C}} \omega\right)_{\bar{P}}$ is a free $\mathcal{O}_{\bar{P}}$-module as expected since $\bar{P}$ is nonsingular. In order to compute $H^{0}(\omega)$ let us check the stalks of $\omega$ at the other (nonsingular) points of $C$. For a point $Q \in C$ given parametrically by $t=c \neq 0$ we have that $t-c$ is the local parameter; we write $x=d t / t^{3}=d(t-c) / t^{3}$, and since $1 / t^{3}$ is a unit in $\mathcal{O}_{Q}$ it is easy to see that $\omega_{Q}=\mathcal{O}_{Q} x$. Finally, at the point $P_{\infty}$ at infinity, the local parameter is $t^{-1}$, so we write $x=-t^{-1} d\left(t^{-1}\right)$. One can check that $V_{x, P_{\infty}}=t \mathcal{O}_{P_{\infty}}$, as we note, for instance, that $t^{2} \notin V_{x, P_{\infty}}$ since $\operatorname{Res}_{P_{\infty}}\left(t^{2} x\right)=\operatorname{Res}_{P_{\infty}}\left(t^{2}\left(-t^{-1} d\left(t^{-1}\right)\right)\right)=\operatorname{Res}_{P_{\infty}}\left(-t d\left(t^{-1}\right)\right)=-1$. If $t^{2} \notin V_{x, P_{\infty}}$ the same holds for all $t^{n}$ for $n \geq 3$, since $V_{x, P_{\infty}}$ is an $\mathcal{O}_{P_{\infty}}$-fractional ideal and $t^{-n} \in \mathcal{O}_{P_{\infty}}$ for all $n \geq 1$. So $\omega_{P_{\infty}}=t \mathcal{O}_{P_{\infty}} x$. Therefore, comparing all stalks, we 
see that $H^{0}(\omega)=\langle x, t x\rangle$ and, as expected, $h^{0}(\omega)=2=g$. It is easily checked that $\widehat{C}=\bar{C}$ in this case. This also stands for an example of a curve satisfying the following definition, which can be easily checked using [2, Prop. 28], for instance.

Definition 2.6. Call $C$ nearly Gorenstein if $C$ has only one non-Gorenstein point $P$ and if the local ring $\mathcal{O}_{P}$ is almost Gorenstein in the sense of Barucci and Fröberg [2. p. 418], namely, if

$$
\operatorname{dim}\left(\overline{\mathcal{O}}_{P} / \mathcal{O}_{P}\right)=\operatorname{dim}\left(\mathcal{O}_{P} / \mathcal{C}_{P}\right)+\operatorname{dim}\left(\operatorname{Ext}^{1}\left(k, \mathcal{O}_{P}\right)\right)-1,
$$

where $k$ is the ground field.

Theorem 2.7. If $C$ is nonhyperelliptic Gorenstein or nearly Gorenstein, then the homomorphisms

$$
\operatorname{Sym}^{n} H^{0}(C, \omega) \longrightarrow H^{0}\left(C, \omega^{n}\right)
$$

are surjective for $n \geq 1$.

Proof. From [5, Prop. 4.5] we have that $\mathcal{O}_{\widehat{C}} \omega$ is an ample invertible sheaf on $\widehat{C}$ which is generated by the pull-back of the sections in $H^{0}(\omega)$. So $\widehat{C}$, originally defined in terms of a sheaf of graded algebras, can actually be written in terms of a regular graded algebra, that is, $\widehat{C}=\operatorname{Proj}\left(\oplus H^{0}\left(\left(\mathcal{O}_{\widehat{C}} \omega\right)^{\otimes n}\right)\right)$. Besides, the complete linear system $\mathrm{L}:=\left|\mathcal{O}_{\widehat{C}} \omega\right|$ is base point free since $H^{0}(\omega) \hookrightarrow H^{0}\left(\mathcal{O}_{\widehat{C}} \omega\right)$. Set $r:=h^{0}\left(\mathcal{O}_{\widehat{C}} \omega\right)-1$ and let $\varphi: \widehat{C} \rightarrow \mathbb{P}^{r}$ be the morphism defined by L. Call $C^{*}:=\varphi(\widehat{C})$. Now $C^{*}=\operatorname{Proj}\left(\oplus H^{0}\left(\mathcal{O}_{C^{*}}(n)\right)\right)$ by the very definition of $C^{*}$. Hence, for every $n \geq 1$, we have a sequence of linear morphisms

$$
\operatorname{Sym}^{n} H^{0}\left(\mathcal{O}_{\widehat{C}} \omega\right) \stackrel{\alpha_{n}}{\longrightarrow} H^{0}\left(\mathcal{O}_{C^{*}}(n)\right) \stackrel{\beta_{n}}{\longrightarrow} H^{0}\left(\left(\mathcal{O}_{\widehat{C}} \omega\right)^{\otimes n}\right),
$$

where $\alpha_{n}$ is the natural homomorphism and $\beta_{n}$ is the injective homomorphism determined by $\varphi$.

If $C$ is Gorenstein, then the $\alpha_{n}$ 's are surjective. In fact, set $d:=\operatorname{deg}\left(C^{*}\right)$. If $C$ is Gorenstein, then $\widehat{C}=C, \mathcal{O}_{\widehat{C}} \omega=\omega ; r=g-1$; the morphism $\varphi$ agrees with the canonical morphism $\kappa: C \rightarrow \mathbb{P}^{g-1}$ and $C^{*}$ is the canonical model in $\mathbb{P}^{g-1}$. Then either $d<2 r$ and hence $C^{*}$ is projectively normal by Lemma 2.2 and hence the $\alpha_{n}$ 's are surjective, or else $d=2 r=2 g-2$. In this case, $\kappa$ is an isomorphism owing to [5, Thm. 4.3]. Hence $\mathcal{O}_{C^{*}}(1)=\omega$ and thus $h^{1}\left(\mathcal{O}_{C^{*}}(1)\right)=1$. Lemma 2.2 now implies that $C^{*}$ is projectively normal and the $\alpha_{n}$ 's are surjective.

If $C$ is Gorenstein and nonhyperelliptic, then the $\beta_{n}$ 's are surjective. In fact, if $C$ is Gorenstein and nonhyperelliptic, then $\varphi$ is an isomorphism owing to [5], Thm. 4.3]. Hence the $\beta_{n}$ 's are surjective.

So the result is proved for Gorenstein nonhyperelliptic curves. Let us prove it for nearly Gorenstein curves.

If $C$ is non-Gorenstein, then the $\alpha_{n}$ 's and the $\beta_{n}$ 's are surjective. In fact, if $C$ is non-Gorenstein, then $\mathcal{O}_{\widehat{C}} \omega$ is very ample owing to [5. Prop. 5.2]. Hence $\varphi$ is an isomorphism and the $\beta_{n}$ 's are surjective. From [5, Prop. 5.2] and its proof we also have $h^{1}\left(\mathcal{O}_{\widehat{C}} \omega\right)=0$ and $\widehat{g} \leq g-2$, where $\widehat{g}$ is the genus of $\widehat{C}$. Thus

$$
\begin{aligned}
d & =\operatorname{deg}_{\widehat{C}}\left(\mathcal{O}_{\widehat{C}} \omega\right)=h^{0}\left(\mathcal{O}_{\widehat{C}} \omega\right)-1+\widehat{g} \\
& =r+\widehat{g} \leq r+g-2=r+h^{0}(\omega)-2 \\
& \leq r+h^{0}\left(\mathcal{O}_{\widehat{C}} \omega\right)-2 \\
& =r+(r+1)-2<2 r .
\end{aligned}
$$


Therefore Lemma 2.2 implies that $C^{*}$ is projectively normal; that is, the $\alpha_{n}$ 's are surjective.

Now take $k(\widehat{C})=k(C)$ and fix a differential $z \in \Omega_{k(C) \mid k}$. Let $P \in C$ and $\widehat{P} \in \widehat{C}$ be over $P$. Since $k(\widehat{C})=k(C)$, we have $\mathcal{O}_{P} \subset \mathcal{O}_{\widehat{P}}$. Write $\omega_{P}=\left(V_{z, P}\right) z:=\{f z \mid f \in$ $\left.V_{z, P}\right\}$, where $V_{z, P}$ is the fractional $\mathcal{O}_{P}$-ideal already defined. Then, keeping the same meaning of notation, we have $\left(\mathcal{O}_{\widehat{C}} \omega\right)_{\widehat{P}}=\left(\mathcal{O}_{\widehat{P}} V_{z, P}\right) z$ and $\left(\widehat{\mathcal{O}}_{\omega}\right)_{P}=\left(\widehat{\mathcal{O}}_{P} V_{z, P}\right) z$, where $\widehat{\mathcal{O}}_{P}=\bigcap_{\widehat{P} \mid P} \mathcal{O}_{\widehat{P}}$. In particular,

$$
\omega_{P} \subset(\widehat{\mathcal{O}} \omega)_{P} \subset\left(\mathcal{O}_{\widehat{C}} \omega\right)_{\widehat{P}}
$$

Since the space of global sections is the intersection of the stalks, we have that $H^{0}(\omega) \subset H^{0}(\widehat{\mathcal{O}} \omega)=H^{0}\left(\mathcal{O}_{\widehat{C}} \omega\right)$. Moreover, in the same way, we also have that $\omega$ is a subsheaf of $\widehat{\mathcal{O}} \omega$, although $\widehat{\mathcal{O}} \omega$ and $\mathcal{O}_{\widehat{C}} \omega$ cannot be compared since they are sheaves on different curves. A similar statement holds for powers; i.e., $H^{0}\left(\omega^{n}\right) \subset$ $H^{0}\left((\widehat{\mathcal{O}} \omega)^{n}\right)=H^{0}\left(\left(\mathcal{O}_{\widehat{C}} \omega\right)^{\otimes n}\right)$, and also $\omega^{n}$ is a subsheaf of $(\widehat{\mathcal{O}} \omega)^{n}$ for all $n \geq 1$ in a natural way. In fact, this can be checked from (44) applied to the stalks in (7) priorly described: one disregards $z$ and just notes that symmetric products of fractional ideals, modded out by torsion, correspond to the regular powers taken in $k(C)$.

Therefore, since $\varphi$ was constructed by the linear series $\left|\mathcal{O}_{\widehat{C}} \omega\right|$, we have that the natural homomorphism, say, $\psi_{n}$ : $\operatorname{Sym}^{n} H^{0}(\omega) \rightarrow H^{0}\left(\omega^{n}\right)$, agrees, for every $n \geq 1$, with the composition $\beta_{n} \circ \alpha_{n}$ restricted to $\operatorname{Sym}^{n} H^{0}(\omega)$. In particular, $\beta_{n} \circ \alpha_{n}\left(\operatorname{Sym}^{n} H^{0}(\omega)\right) \subset H^{0}\left(\omega^{n}\right) \subset H^{0}\left(\left(\mathcal{O}_{\widehat{C}} \omega\right)^{\otimes n}\right)$ for all $n \geq 1$. So if $H^{0}(\omega)=$ $H^{0}\left(\mathcal{O}_{\widehat{C}} \omega\right)$, then $\beta_{n} \circ \alpha_{n}$ and $\psi_{n}$ coincide because they have the same source.

Now assume $C$ is nearly Gorenstein. If so, $H^{0}(\omega)=H^{0}\left(\mathcal{O}_{\widehat{C}} \omega\right)$ due to 5 , Lem. 5.8]. Thus $\psi_{n}=\beta_{n} \circ \alpha_{n}$ for each $n \geq 1$. Besides, $C$ is also non-Gorenstein, but we have just proved that the $\beta_{n} \circ \alpha_{n}$ are surjective in this case, so the theorem follows.

Remark 2.8. The last lines of the proof above imply that if $C$ is nearly Gorenstein, then $H^{0}\left(\omega^{n}\right)=H^{0}\left(\left(\mathcal{O}_{\widehat{C}} \omega\right)^{\otimes n}\right)$ for every $n \geq 1$. In fact, $\beta_{n} \circ \alpha_{n}$ surjects $H^{0}\left(\left(\mathcal{O}_{\widehat{C}} \omega\right)^{\otimes n}\right)$ and $\psi_{n}\left(\operatorname{Sym}^{n} H^{0}(\omega)\right) \subset H^{0}\left(\omega^{n}\right)$, so if $\beta_{n} \circ \alpha_{n}=\psi_{n}$ the assertion follows. Using Definition 2.6 we are able to state more: we claim that actually $\omega^{n}=(\widehat{\mathcal{O}} \omega)^{n}$ for $n \geq 2$. Indeed, if $C$ is nearly Gorenstein, then $C$ has just one non-Gorenstein point, say $P$, for which the local ring $\mathcal{O}_{P}$ is almost Gorenstein. Moreover, from [2, Prop. 28] we have that if $\mathcal{O}_{P}$ is almost Gorenstein, then $\operatorname{dim}\left((\widehat{\mathcal{O}} \omega)_{P} / \omega_{P}\right)=1$. Now take $x$ as in Definition 2.4. If so, $(\widehat{\mathcal{O}} \omega)_{P}=\widehat{\mathcal{O}}_{P} x$ [5. proof of Prop. 5.2] and hence $\mathcal{O}_{P} \subset V_{x, P} \subset \widehat{\mathcal{O}}_{P}$ by the very definition of $\widehat{C}$. Then $V_{x, P}^{2} \subset \widehat{\mathcal{O}}_{P}$, but $V_{x, P} \subsetneq V_{x, P}^{2}$ owing to the proof of [2, Prop. 28]. Hence $V_{x, P}^{2}=\widehat{\mathcal{O}}_{P}$ because $\operatorname{dim}\left(\widehat{\mathcal{O}}_{P} / V_{x, P}\right)=\operatorname{dim}\left((\widehat{\mathcal{O}} \omega)_{P} / \omega_{P}\right)=1$. Therefore $V_{x, P}^{n}=\widehat{\mathcal{O}}_{P}=\widehat{\mathcal{O}}_{P}^{n}$ for $n \geq 2$. This yields $\omega_{P}^{n}=\left((\widehat{\mathcal{O}} \omega)^{n}\right)_{P}$ for $n \geq 2$. Since $P$ is the only non-Gorenstein point of $C$, then $\omega$ and $\widehat{\mathcal{O}} \omega$ agree outside $P$. Thus $\omega^{n}=(\widehat{\mathcal{O}} \omega)^{n}$ for $n \geq 2$, and the claim is proved.

Definition 2.9. In [7, p. 188 top] Rosenlicht showed that the linear system $\overline{\mathrm{L}}:=$ $\left(\mathcal{O}_{\bar{C}} \omega, H^{0}(\omega)\right)$ is base point free. He then considered the morphism $\bar{\kappa}: \bar{C} \rightarrow \mathbb{P}^{g-1}$ defined by $\overline{\mathrm{L}}$ and called $C^{\prime}:=\bar{\kappa}(C)$ the canonical model of $C$. In [5, Dfn. 4.9] one finds another characterization of $C^{\prime}$. It is the image of the morphism $\widehat{\kappa}: \widehat{C} \rightarrow \mathbb{P}^{g-1}$ defined by the linear system $\widehat{\mathrm{L}}:=\left(\mathcal{O}_{\widehat{C}} \omega, H^{0}(\omega)\right)$. Note that this definition of 
$C^{\prime}$ agrees with the former, which appears in the Introduction. In fact, if $C$ is Gorenstein, then $\widehat{C}=C, \mathcal{O}_{\widehat{C}} \omega=\omega$ and $\widehat{\kappa}$ is nothing but $\kappa$, the canonical morphism.

Remark 2.10. As pointed out in the Introduction, Max Noether's assertion implies Rosenlicht's. In fact, since $\omega$ is generated by global sections, Rosenlicht's assertion is equivalent to this one: $\widehat{\kappa}: \widehat{C} \rightarrow C^{\prime}$ is an isomorphism. So assume $C$ is nonhyperelliptic and assume Max Noether's assertion holds; that is, $\operatorname{Sym}^{n} H^{0}(\omega) \rightarrow H^{0}\left(\omega^{n}\right)$ is surjective for $n \geq 1$. We will show that $\widehat{\kappa}$ is an isomorphism. We first claim there exists $n$ such that $\omega^{n}=(\widehat{\mathcal{O}} \omega)^{n}$. Indeed, for every singular point $P \in C$, it follows that $\mathcal{O}_{P} \subset V_{x, P} \subset \widehat{\mathcal{O}}_{P}$. Consider the sequence

$$
V_{x, P} \subset V_{x, P}^{2} \subset \ldots \subset V_{x, P}^{i} \subset \ldots \subset \widehat{\mathcal{O}}_{P} .
$$

There exists $n$, depending on $P$, such that $V_{x, P}^{i}=V_{x, P}^{n}$ for every $i \geq n$ because $\operatorname{dim}\left(\widehat{\mathcal{O}}_{P} / V_{x, P}\right)<\infty$. Now $V_{x, P}^{n}$ is a ring and $\widehat{\mathcal{O}}_{P}$ is the smallest ring in $k(C)$ that contains $V_{x, P}$. Thus $V_{x, P}^{n}$ and $\widehat{\mathcal{O}}_{P}$ agree and so do $\omega_{P}^{n}$ and $(\widehat{\mathcal{O}} \omega)^{n}$. Take an $n$ that works for all singular points of $C$. Since $\omega$ and $\widehat{\mathcal{O}} \omega$ agree outside the singular points of $C$, we have $(\widehat{\mathcal{O}} \omega)^{n}=\omega^{n}$. For this $n$, consider the sequence

$$
\operatorname{Sym}^{n} H^{0}(\omega) \stackrel{\alpha}{\longrightarrow} H^{0}\left(\mathcal{O}_{C^{\prime}}(n)\right) \stackrel{\beta}{\longrightarrow} H^{0}\left(\left(\mathcal{O}_{\widehat{C}} \omega\right)^{\otimes n}\right)=H^{0}\left((\widehat{\mathcal{O}} \omega)^{n}\right)=H^{0}\left(\omega^{n}\right),
$$

where $\alpha$ and $\beta$ are naturally associated to the morphism $\widehat{\kappa}: \widehat{C} \rightarrow C^{\prime}$. Max Noether's assertion implies that $\beta \circ \alpha$ is surjective, so $\beta$ is surjective. But $\beta$ is injective, so $\beta$ is bijective. Hence $h^{0}\left(\mathcal{O}_{C^{\prime}}(n)\right)=h^{0}\left(\left(\mathcal{O}_{\widehat{C}} \omega\right)^{\otimes n}\right)$. If $C$ is nonhyperelliptic, then $\operatorname{deg}_{C^{\prime}}\left(\mathcal{O}_{C^{\prime}}(n)\right)=\operatorname{deg}_{\widehat{C}}\left(\left(\mathcal{O}_{\widehat{C}} \omega\right)^{\otimes n}\right)$. Besides, $h^{1}\left(\mathcal{O}_{C^{\prime}}(n)\right)=h^{1}\left(\left(\mathcal{O}_{\widehat{C}} \omega\right)^{\otimes n}\right)=0$ taking $n \gg 0$. Hence the Riemann-Roch theorem implies that $\widehat{C}$ and $C^{\prime}$ are of the same arithmetic genus. Therefore, $\widehat{\kappa}: \widehat{C} \rightarrow C^{\prime}$ is an isomorphism; in other words, Rosenlicht's assertion holds.

\section{Generalization by means of an intrinsic argument (main theorem)}

In this section we prove the main theorem announced in the Introduction: that is, Max Noether's assertion holds for nonhyperelliptic curves for which the nonGorenstein singularities satisfy the following property:

Definition 3.1. A point $P \in C$ is called unibranch if $\nu^{-1}(P)$ consists of only one point.

For the remainder, we recall some useful results on local algebra and the semigroup of values. As in ([9, p. 113], for instance), the conductor $\mathcal{C}$ is defined as the coherent fractional ideal sheaf on $C$ such that, for each $P \in C$, we have $\mathcal{C}_{P}=\left(\mathcal{O}_{P}: \overline{\mathcal{O}}_{P}\right)$, the largest $\overline{\mathcal{O}}_{P}$-ideal contained in $\mathcal{O}_{P} ;$ in particular, $\mathcal{C}_{P}$ is an $\mathcal{O}_{P^{-}}$ ideal as well. Now assume $P$ is singular. From our choice of $x \in \Omega_{k(C) \mid k}$, as in Definition 2.4, we have already seen in the proof of Theorem 2.7 that $\mathcal{O}_{P} \subset V_{x, P} \subset \widehat{\mathcal{O}}_{P}$. So we have a long sequence of inclusions of the $\mathcal{O}_{P}$-ideals that we are especially interested in:

$\overline{\mathcal{O}}_{P} \supset \widehat{\mathcal{O}}_{P} \supset \ldots \supset V_{x, P}^{n} \supset \ldots \supset V_{x, P}^{2} \supset V_{x, P} \supset \mathcal{O}_{P} \supset \mathfrak{m}_{P} \supset \mathcal{C}_{P} \supset \mathcal{C}_{P}^{2} \supset \ldots \supset \mathcal{C}_{P}^{n} \ldots$, where $\mathfrak{m}_{P}$ is the maximal ideal of $\mathcal{O}_{P}$.

Now assume $P$ is unibranch. We use the same notation $v_{P}$ to the valuation of $\overline{\mathcal{O}}_{P}$ applied to either rational functions or differentials. We clearly have $v_{P}\left(\overline{\mathcal{O}}_{P}\right)=\mathbb{N}$. 
Set $\mathrm{S}_{P}:=v_{P}\left(\mathcal{O}_{P}\right)$. Define $\mathrm{M}_{P}:=\mathrm{S}_{P} \backslash 0$ and $\mathrm{C}_{P}:=\left\{a \in \mathbb{Z} \mid a+\mathbb{N} \subset \mathrm{S}_{P}\right\}$. We feature two elements in $\mathrm{S}_{P}$ which will be important later on:

$$
\alpha_{P}:=\min \left(\mathrm{C}_{P}\right) \quad \text { and } \quad \beta_{P}:=\min \left(\mathrm{M}_{P}\right) \text {. }
$$

By definition, $\alpha_{P}-1$ is the largest integer not in $S_{P}$. From [6, Lem. 2], we have $\mathcal{C}_{P}=\left\{f \in k(C) \mid v_{P}(f) \geq \alpha_{P}\right\}$. So we also have $\mathcal{C}_{P}=t_{P}^{\alpha_{P}} \overline{\mathcal{O}}_{P}$, where $t_{P}$ is a local parameter in $\overline{\mathcal{O}}_{P}$. Note also that $\beta_{P}$ agrees with the multiplicity of $P$. It is easily seen that $v_{P}\left(\mathfrak{m}_{P}\right)=\mathrm{M}_{P}$. Moreover, since the $\overline{\mathcal{O}}_{P}$-ideals are of the form $t_{P}^{a} \overline{\mathcal{O}}_{P}$ and the latter agrees with $\left\{f \in k(C) \mid v_{P}(f) \geq a\right\}$, it follows that $t_{P}^{\beta_{P}} \overline{\mathcal{O}}_{P}$ is the smallest $\overline{\mathcal{O}}_{P}$-ideal which contains $\mathfrak{m}_{P}$, that is, $\mathfrak{m}_{P} \overline{\mathcal{O}}_{P}=t_{P}^{\beta_{P}} \overline{\mathcal{O}}_{P}$.

From [9, Thm. 2.11] we have

$$
v_{P}\left(\omega_{P}\right)=\left\{a \in \mathbb{Z} \mid-a-1 \notin \mathrm{S}_{P}\right\} .
$$

Now $\bar{\omega}_{P}=\mathcal{C}_{P} x$ owing to [5, Lem. 2.8] and $v_{P}\left(\bar{\omega}_{P}\right)=\mathbb{N}$ owing to [9, Thm. 2.12] applied to a nonsingular point. This implies that $v_{P}(x)=-\alpha_{P}$, which yields

$$
v\left(V_{x, P}\right)=\mathrm{K}_{P}:=\left\{a \in \mathbb{Z} \mid \alpha_{P}-a-1 \notin \mathrm{S}_{P}\right\} .
$$

So we also have a sequence of inclusions in $\mathbb{Z}$ closely related to some $\mathcal{O}_{P}$-ideals we are focused on:

$$
\mathbb{N} \supset \mathrm{K}_{P} \supset \mathrm{S}_{P} \supset \mathrm{M}_{P} \supset \mathrm{C}_{P} .
$$

We claim that the point $P$ is Gorenstein if and only if $\mathrm{K}_{P}=\mathrm{S}_{P}$. In fact, we have that $P$ is Gorenstein if and only if $V_{x, P}=\mathcal{O}_{P}$ owing to [5, Lem. 2.9, Eq. (2.9.2)]; on the other hand, $\operatorname{dim}\left(V_{x, P} / \mathcal{O}_{P}\right)=\#\left(\mathrm{~K}_{P} \backslash \mathrm{S}_{P}\right)$ owing to [6, Prop. 1]. The claim follows. Actually, the claim corresponds to another one which appears frequently in the literature related to semigroups of one-dimensional local rings, even in the multibranch case: the ring is Gorenstein if and only if its semigroup is symmetric (cf. 2, 3] and the references therein).

Lemma 3.2. Let $P \in C$ be a unibranch non-Gorenstein point and $t_{P}$ a local parameter in $\overline{\mathcal{O}}_{P}$. Then

$$
W_{x}^{n} \longrightarrow V_{x, P}^{n} / t_{P}^{-\epsilon} \mathcal{C}_{P}^{n}
$$

is surjective for every $n \geq 2$ in the following cases:

(i) $\epsilon=2 n-1$;

(ii) $\epsilon=1$ if there is $y_{0} \in H^{0}(\omega)$ with $v_{P}\left(y_{0}\right)=0$;

(iii) $\epsilon=0$ if there are $y_{0}, y_{1} \in H^{0}(\omega)$ with $v_{P}\left(y_{0}\right)=0$, and $v_{P}\left(y_{1}\right)=1$ or 2 .

Proof. Call $\alpha:=\alpha_{P}, \beta:=\beta_{P}$ and $t:=t_{P}$ for short. Consider the sequence

$$
V_{x, P}^{n} \supset \mathcal{C}_{P} \supset t^{\alpha-\beta} \mathcal{C}_{P}=t^{-\beta} \mathcal{C}_{P}^{2} \supset \mathcal{C}_{P}^{2} \supset \mathcal{C}_{P}^{n}
$$

of which each inclusion will correspond to a step of our proof.

Step 1. $W_{x}^{n} \rightarrow V_{x, P}^{n} / \mathcal{C}_{P}$.

From the proof of [5. Lem. 6.1] we have $V_{x, P} \subset W_{x}+\mathcal{C}_{P}$. On the other hand, both $\mathcal{C}_{P}$ and $W_{x}$ are contained in $V_{x, P}$. We are led to

$$
V_{x, P}=W_{x}+\mathcal{C}_{P} .
$$

Now $W_{x} \subset V_{x, P} \subset \overline{\mathcal{O}}_{P}$. Thus if $a \in W_{x}$ and $b \in \mathcal{C}_{P}$, then $a b \in \mathcal{C}_{P}$. This yields

$$
V_{x, P}^{n}=W_{x}^{n}+\mathcal{C}_{P},
$$

which proves the claim of the first step. 
Step 2. $\mathcal{C}_{P} / t^{\alpha-\beta} \mathcal{C}_{P}$ is generated by images of elements in $W_{x}^{2}$.

Call $v:=v_{P}, \mathrm{~S}:=\mathrm{S}_{P}$ and $\mathrm{K}:=\mathrm{K}_{P}$ for short. Since $P$ is non-Gorenstein, $\mathrm{K} \supsetneq \mathrm{S}$. Take $d_{1} \in \mathrm{K} \backslash \mathrm{S}$. Then $d_{2}:=\alpha-d_{1}-1 \in \mathrm{K} \backslash \mathrm{S}$ by the very definition of $\mathrm{K}$.

Let $r$ be the greatest integer such that $(r+1) \beta \leq \alpha$. Assume $r \geq 1$. In particular, $2 \beta \leq \alpha$. We claim that for every $1 \leq i \leq r$ one can find nonnegative integers $q_{i 1}, q_{i 2}$ such that $i=q_{i 1}+q_{i 2}$ and $q_{i j} \beta+d j<\alpha$ for $j=1,2$. Indeed, it suffices to prove this for $r$ because if $i<r$, one can take $q_{i 1}:=\min \left\{i, q_{r 1}\right\}$ and $q_{i 2}:=i-q_{i 1} \leq q_{r 2}$. Suppose without loss of generality that $d_{1} \leq d_{2}$. Take $q_{r 2}$ as the greatest integer such that $q_{r 2} \beta \leq d_{1}$ and $q_{r 1}:=r-q_{r 2}$. Since $d_{1} \leq d_{2}$ and $d_{1}+d_{2}=\alpha-1$ we have $2 d_{1}<\alpha$. Therefore $\left(q_{r 2}+1\right) \beta \leq d_{1}+\beta \leq 2 \max \left\{d_{1}, \beta\right\} \leq \alpha$ and hence $r \geq q_{r 2}$ due to the definition of $r$. This implies that $q_{r 1} \geq 0$. Now $q_{r 2} \beta+d_{2} \leq d_{1}+d_{2}<\alpha$ and $q_{r 1} \beta+d_{1}=\left(r-q_{r 2}\right) \beta+d_{1}=r \beta-q_{r 2} \beta+d_{1} \leq(\alpha-\beta)+\left(d_{1}-q_{r 2} \beta\right)<\alpha$ because $d_{1}-q_{r 2} \beta<\beta$ due to the definition of $q_{r 2}$. This proves the claim.

Now take $a_{1}, a_{2} \in V_{x, P}$ such that $v\left(a_{1}\right)=d_{1}$ and $v\left(a_{2}\right)=d_{2}$, and $m \in \mathcal{O}_{P}$ such that $v(m)=\beta$. Since $V_{x, P}$ is an $\mathcal{O}_{P}$-module, $m^{q_{i j}} a_{j} \in V_{x, P}$ for $1 \leq i \leq r$ and $j=1$,2. From (10), write $m^{q_{i j}} a_{j}=a_{i j}^{\prime}+a_{i j}^{\prime \prime}$ with $a_{i j}^{\prime} \in W_{x}$ and $a_{i j}^{\prime \prime} \in \mathcal{C}_{P}$. Set $f_{i}:=a_{i 1}^{\prime} a_{i 2}^{\prime}$. We have $f_{i} \in W_{x}^{2}$ for $1 \leq i \leq r$. Besides,

$$
\begin{aligned}
v\left(f_{i}\right) & =v\left(a_{i 1}^{\prime}\right)+v\left(a_{i 2}^{\prime}\right)=v\left(m^{q_{i 1}} a_{1}\right)+v\left(m^{q_{i 2}} a_{2}\right) \\
& =v\left(m^{q_{i 1}+q_{i 2}} a_{1} a_{2}\right)=v\left(m^{i} a_{1} a_{2}\right) \\
& =i \beta+d_{1}+d_{2}=i \beta+\alpha-1,
\end{aligned}
$$

where the second equality holds because, for $j=1,2$, we have $v\left(m^{q_{i j}} a_{j}\right)=q_{i j} \beta+$ $d_{j}<\alpha \leq v\left(a_{i j}^{\prime \prime}\right)$, which implies that $v\left(m^{q_{i j}} a_{j}\right)=v\left(a_{i j}^{\prime}\right)$.

We claim that one can find a set of elements $\left\{b_{1}, \ldots, b_{\beta-1}\right\} \subset W_{x}$ such that

$$
v\left(b_{i}\right)=i+\alpha-\beta-1
$$

in fact, from (9), we have $i+\alpha-\beta-1 \in \mathrm{K}$ because $0<\alpha-(i+\alpha-\beta-1)-1=$ $\beta-i<\beta$ and hence $\alpha-(i+\alpha-\beta-1)-1 \notin \mathrm{S}$. Thus there is $b_{i}^{\prime} \in V_{x, P}$ such that $v\left(b_{i}^{\prime}\right)=i+\alpha-\beta-1$ : write $b_{i}^{\prime}=b_{i}+c_{i}$ as in (10) with $b_{i} \in W_{x}$ and $c_{i} \in \mathcal{C}_{P}$. Since $v\left(c_{i}\right) \geq \alpha>i+\alpha-\beta-1=v\left(b_{i}^{\prime}\right)$, we must have $v\left(b_{i}^{\prime}\right)=v\left(b_{i}\right)$, and the claim follows. Now for $1 \leq i \leq r$ we clearly have $m^{i} \in \mathcal{O}_{P} \subset V_{x, P}$ and we also have $v\left(m^{i}\right)=i \beta<\alpha$. Use (10) to find $m_{i} \in W_{x}$ such that $v\left(m_{i}\right)=i \beta$. If $\beta \nmid \alpha$ we claim that there is $m_{r+1} \in W_{x}$ such that $v\left(m_{r+1}\right)=(r+1) \beta$. In fact, in this case, $\alpha-(r+1) \beta$ cannot be 0 since $\beta \nmid \alpha$, cannot be 1 since $\alpha-1$ is never in $\mathrm{S}$ and $(r+1) \alpha$ is always in $\mathrm{S}$, and cannot be smaller than 0 or larger than or equal to $\beta$ due to the definition of $r$. So $2 \leq \alpha-(r+1) \beta<\beta$, which implies $1 \leq \alpha-(r+1) \beta-1<\beta$ and hence $\alpha-(r+1) \beta-1 \notin \mathrm{S}$ because $\beta$ is its smallest strictly positive element. It follows that $(r+1) \beta \in \mathrm{K}$, and one finds such an $m_{r+1}$ the same way as above.

Now write $\alpha=(r+1) \beta+p$ with $0 \leq p<\beta$. Set

$$
A_{1}:=\left(\bigcup_{i=1}^{r}\left\{m_{i} b_{1}, \ldots, m_{i} b_{\beta-1}, f_{i}\right\}\right) \cup\left\{m_{r+1} b_{1}, \ldots, m_{r+1} b_{p}\right\},
$$

where the set at the left hand side of the union is empty if $r=0$ and so is the set at the right hand side if $p=0$. We claim that $A_{1}$, which is contained in $W_{x}^{2}$ by construction, provides a basis for $\mathcal{C}_{P} / t^{\alpha-\beta} \mathcal{C}_{P}$. In fact, first, $A_{1}$ is of the right size because $\operatorname{dim}\left(\mathcal{C}_{P} / t^{\alpha-\beta} \mathcal{C}_{P}\right)=\alpha-\beta$ and $\left|A_{1}\right|=r \beta+p=\alpha-\beta$. Second, $A_{1} \subset \mathcal{C}_{P}$ and its elements are linearly independent $\bmod t^{\alpha-\beta} \mathcal{C}_{P}$, as one can see by computing 
values:

$$
\begin{array}{cccc}
v\left(m_{1} b_{1}\right)=\alpha & \ldots & v\left(m_{1} b_{\beta-1}\right)=\alpha+\beta-2 & v\left(f_{1}\right)=\alpha+\beta-1 \\
\vdots & & \vdots & \vdots \\
v\left(m_{r} b_{1}\right)=\alpha+(r-1) \beta & \ldots & v\left(m_{r} b_{\beta-1}\right)=\alpha+r \beta-2 & v\left(f_{r}\right)=\alpha+r \beta-1 \\
v\left(m_{r+1} b_{1}\right)=\alpha+r \beta & \ldots & v\left(m_{r+1} b_{p}\right)=2 \alpha-\beta-1 . &
\end{array}
$$

This proves the claim, and we are done with the second step.

Step 3.1. $t^{-\beta} \mathcal{C}_{P}^{2} / t^{-3} \mathcal{C}_{P}^{2}$ is generated by images of elements in $W_{x}^{2}$.

Assume $\beta>3$ for otherwise the claim trivially holds. Consider the set

$$
A_{2}:=\left\{b_{\beta-1} b_{3}, \ldots, b_{\beta-1} b_{\beta-2}, b_{\beta-1}^{2}\right\} .
$$

It is contained in $W_{x}^{2}$ by construction and provides a basis for $t^{-\beta} \mathcal{C}_{P}^{2} / t^{-3} \mathcal{C}_{P}^{2}$. In fact, $\operatorname{dim}\left(t^{-\beta} \mathcal{C}_{P}^{2} / t^{-3} \mathcal{C}_{P}^{2}\right)=\beta-3=\left|A_{2}\right|$. Besides, $A_{2} \subset t^{-\beta} \mathcal{C}_{P}^{2}$ and its elements are linearly independent $\bmod t^{-3} \mathcal{C}_{P}^{2}$ as one can see by computing values:

$$
v\left(b_{\beta-1} b_{3}\right)=2 \alpha-\beta \quad \ldots \quad v\left(b_{\beta-1}^{2}\right)=2 \alpha-4 .
$$

Step 3.2. If (ii) or (iii) holds, then $t^{-\beta} \mathcal{C}_{P}^{2} / t^{-\epsilon} \mathcal{C}_{P}^{2}$ is generated by images of elements in $W_{x}^{2}$.

If there is $y_{0} \in H^{0}(\omega)$ with $v\left(y_{0}\right)=0$, then $h_{0}:=y_{0} / x \in W_{x}$ and $v\left(h_{0}\right)=\alpha$. Consider the set

$$
A_{2}^{\prime}:=\left\{h_{0} b_{1}, \ldots, h_{0} b_{\beta-1}\right\} .
$$

It is contained in $W_{x}^{2}$ by construction and provides a basis for $t^{-\beta} \mathcal{C}_{P}^{2} / t^{-1} \mathcal{C}_{P}^{2}$. In fact, $\operatorname{dim}\left(t^{-\beta} \mathcal{C}_{P}^{2} / t^{-1} \mathcal{C}_{P}^{2}\right)=\beta-1=\left|A_{2}^{\prime}\right|$. Besides, $A_{2} \subset t^{-\beta} \mathcal{C}_{P}^{2}$ and its elements are linearly independent $\bmod t^{-1} \mathcal{C}_{P}^{2}$, as one can see by computing values:

$$
v\left(h_{0} b_{1}\right)=2 \alpha-\beta \quad \ldots \quad v\left(h_{0} b_{\beta-1}\right)=2 \alpha-2 .
$$

If, besides, there is $y_{1} \in H^{0}(\omega)$ such that $v\left(y_{1}\right)=1$ (resp. $v\left(y_{1}\right)=2$ ), then $h_{1}:=y_{1} / x \in W_{x}$ and $v\left(h_{1}\right)=\alpha+1$ (resp. $v\left(h_{1}\right)=\alpha+2$ ). Thus one forms $A_{2}^{\prime \prime}$ by adjoining $h_{1} b_{\beta-1}$ (resp. $h_{1} b_{\beta-2}$ ) to $A_{2}^{\prime}$ in order to get a basis for $t^{-\beta} \mathcal{C}_{P}^{2} / \mathcal{C}_{P}^{2}$.

Step 3.3. $t^{-3} \mathcal{C}_{P}^{2} / t^{-2 n+1} \mathcal{C}_{P}^{n}$ is generated by images of elements in $W_{x}^{n}$.

The set $B:=\left\{f_{1}\right\} \cup A_{1} \cup A_{2}$ is contained in $W_{x}^{2}$ by construction. Then

$$
A_{3}:=\bigcup_{i=1}^{n-2} b_{\beta-1}^{i} B
$$

and $A_{3} \subset t^{-3} \mathcal{C}_{P}^{2}$ and its elements are linearly independent $\bmod t^{-2 n+1} \mathcal{C}_{P}^{n}$, as one sees by computing values:

$$
v\left(b_{\beta-1} f_{1}\right)=2 \alpha-3 \quad \ldots \quad v\left(b_{\beta-1}^{n}\right)=n \alpha-2 n .
$$

Step 3.4. If (ii) or (iii) holds, then $t^{-\epsilon} \mathcal{C}_{P}^{2} / t^{-\epsilon} \mathcal{C}_{P}^{n}$ is generated by images of elements in $W_{x}^{n}$.

The set $B^{\prime}:=\left\{f_{1}\right\} \cup A_{1} \cup A_{2}^{\prime}$ is contained in $W_{x}^{2}$ by construction. Then

$$
A_{3}^{\prime}:=\bigcup_{i=1}^{n-2} h_{0}^{i} B^{\prime}
$$


is contained in $W_{x}^{n}$, and it is easily checked that $A_{3} \subset t^{-1} \mathcal{C}_{P}^{2}$ and its elements are linearly independent $\bmod t^{-1} \mathcal{C}_{P}^{n}$. If there is $y_{1} \in H^{0}(\omega)$ such that $v\left(y_{1}\right)=1$ or 2 , then form $B^{\prime \prime}$ by discarding $f_{1}$ in $B^{\prime}$ and replacing $A_{2}^{\prime}$ by $A_{2}^{\prime \prime}$. Then form $A_{3}^{\prime \prime}$ by replacing $B^{\prime}$ by $B^{\prime \prime}$ in $A_{3}^{\prime}$. We are finally done.

Lemma 3.3. Let $P \in C$ be a nonsingular point. Then:

(i) If $g \geq 1$, there is $y_{0} \in H^{0}(\omega)$ with $v_{P}\left(y_{0}\right)=0$.

(ii) If $C$ is nonhyperelliptic, there is $y_{1} \in H^{0}(\omega)$ with $v_{P}\left(y_{1}\right)=1$.

(iii) If $C$ is hyperelliptic and $g \geq 2$, there is $y_{1} \in H^{0}(\omega)$ with $v_{P}\left(y_{1}\right)=1$ or 2 .

Proof. According to [4, p. $536 \mathrm{mid}$ ] or [5, Lem 3.1.(1)], if $g \geq 1$, then $\omega$ is generated by global sections; in particular $\omega_{P}=\mathcal{O}_{P} y_{0}$ for $y_{0} \in H^{0}(\omega)$. Since $P$ is nonsingular, from (8) there is $y \in \omega_{P}$ such that $v_{P}(y)=0$. We have $v_{P}\left(y_{0}\right) \geq 0$ due to (8) as well. Since $y=f y_{0}$ for $f \in \mathcal{O}_{P}$, this forces $v_{P}\left(y_{0}\right)=0$ and (i) is proved.

Assume that $C$ is nonhyperelliptic. If $h^{0}(\omega(-P))=h^{0}(\omega(-2 P))$, then $h^{1}(\omega(-2 P))>h^{1}(\omega(-P))$ because $\operatorname{deg}(\omega(-P))>\operatorname{deg}(\omega(-2 P))$. So $h^{0}(\mathcal{O}(2 P))>$ $h^{0}(\mathcal{O}(P)) \geq 1$. Since $\operatorname{deg}(\mathcal{O}(2 P))=2$, the existence of a degree 2 morphism $C \rightarrow \mathbb{P}^{1}$ follows, contrary to the hypothesis that $C$ is nonhyperelliptic. Therefore $h^{0}(\omega(-P))>h^{0}(\omega(-2 P))$. Thus there exists $y_{1} \in H^{0}(\omega(-P)) \subset H^{0}(\omega)$ such that $v\left(y_{1}\right)=1$ and (ii) is proved.

Assume that $C$ is hyperelliptic and that $g \geq 2$. According to [10, pp. 95-96], [7. p. 188 top] or [5, Prop. 2.6(1)] there is $a \in k(C)$ and a differential $y$ such that $[k(C): k(a)]=2$ and $H^{0}(\omega)=\left\langle y, a y, \ldots, a^{g-1} y\right\rangle$, and we may also assume $\operatorname{div}_{0}(a)=P+Q$. Again, since $\omega$ is generated by $H^{0}(\omega)$ and since $v_{P}(a) \geq 1$, one has $\omega_{P}=\mathcal{O}_{P} y$ which forces $v_{P}(y)=0$. Setting $y_{1}:=b y$, then $v_{P}\left(y_{1}\right)=1$ or 2 depending on whether $P$ differs from $Q$ or not. This proves (iii).

Lemma 3.4. If $g \geq 2$, then $h^{1}\left(\omega^{n}\right)=0$ for $n \geq 2$.

Proof. We first claim that $\operatorname{deg}\left(\omega^{n}\right)>\operatorname{deg}(\omega)$ for every $n \geq 2$. In fact, set $\eta_{P}=$ $\operatorname{dim}\left(\omega_{P} / \mathcal{O}_{P} x\right)$ and $\eta=\sum_{P \in C} \eta_{P}$. Then, focusing just on the contributions of Gorenstein points to $\operatorname{deg}(\omega)$, we get

$$
\begin{aligned}
\operatorname{deg}\left(\omega^{n}\right)-\operatorname{deg}(\omega) & \geq(n-1)(2 g-2-\eta) \\
& =(n-1)((g-2)+(g-\eta)) .
\end{aligned}
$$

But $g>\eta$ unless $g=0$ owing to the proof of [5, Prop. 5.2]. This proves the claim. Therefore $\chi\left(\omega^{n}\right)>\chi(\omega)=g-1$, and the result follows from [5, Lem. 5.1.(1)].

Lemma 3.5. Let $P \in C$ be a unibranch non-Gorenstein point. Let $\widetilde{C}$ be the curve obtained from $C$ resolving $P$ and $\widetilde{g}$ its genus. Let $\varphi: \widetilde{C} \rightarrow C$ be the natural morphism and $\widetilde{\omega}:=\varphi_{*}\left(\omega_{\widetilde{C}}\right)$. If $\widetilde{g} \geq 2$, then

$$
\operatorname{Sym}^{n} H^{0}(\omega) \longrightarrow H^{0}\left(\omega^{n}\right) / H^{0}\left(\widetilde{\omega}^{n}\right)
$$

is surjective for every $n \geq 1$.

Proof. Note first that once a differential is fixed, the stalk of the dualizing sheaf of any curve at a point is completely determined by its local ring. So if we take $k(\widetilde{C})=k(\bar{C})=k(C)$, we clearly have $\widetilde{\omega}_{Q}=\omega_{Q}$ for $Q \neq P$ and $\widetilde{\omega}_{P}=\bar{\omega}_{P}=\mathcal{C}_{P} \omega_{P} \subset$ $\omega_{P}$ (see [5, Lem. 2.8] for the second equality). So we have that $\widetilde{\omega}^{n}$ is a subsheaf of $\omega^{n}$ for all $n \geq 1$. 
Form the long exact sequence

$$
0 \rightarrow H^{0}\left(\widetilde{\omega}^{n}\right) \rightarrow H^{0}\left(\omega^{n}\right) \stackrel{u}{\rightarrow} H^{0}\left(\omega^{n} / \widetilde{\omega}^{n}\right) \rightarrow H^{1}\left(\widetilde{\omega}^{n}\right) \rightarrow H^{1}\left(\omega^{n}\right) .
$$

Now $h^{1}(\omega)=h^{1}(\widetilde{\omega})=1$ and $g>\widetilde{g} \geq 2$, which implies $h^{1}\left(\omega^{n}\right)=h^{1}\left(\widetilde{\omega}^{n}\right)=0$ for $n \geq 2$ due to Lemma 3.4. Then $u$ is surjective, and so $H^{0}\left(\omega^{n}\right) / H^{0}\left(\widetilde{\omega}^{n}\right)=$ $H^{0}\left(\omega^{n} / \widetilde{\omega}^{n}\right)=\omega_{P}^{n} / \widetilde{\omega}_{P}^{n}$. So we need to show that $\operatorname{Sym}^{n} H^{0}(\omega) \rightarrow \omega_{P}^{n} / \widetilde{\omega}_{P}^{n}$ is surjective.

As said above, $\widetilde{\omega}_{P}=\bar{\omega}_{P}$, and $\bar{\omega}_{P}=\mathcal{C}_{P} x$ owing also to [5, Lem. 2.8]. This implies $\widetilde{V}_{x, P}=\mathcal{C}_{P}$. Now $\omega_{P}^{n} / \widetilde{\omega}_{P}^{n}=V_{x, P}^{n} / \widetilde{V}_{x, P}^{n}=V_{x, P}^{n} / \mathcal{C}_{P}^{n}$. Besides, $\operatorname{Sym}^{n} H^{0}(\omega) \rightarrow W_{x}^{n}$ is surjective, as can be seen by considering the map $H^{0}(\omega) \rightarrow W_{x}$ given by $y \rightarrow y / x$ and then taking $n$-fold products in $k(C)$. So it suffices to show that $W_{x}^{n} \rightarrow V_{x, P}^{n} / \mathcal{C}_{P}^{n}$ is surjective for $n \geq 1$.

Let $\widetilde{P}$ be the point of $\widetilde{C}$ which lies over $P$. Since $\widetilde{g} \geq 2$, Lemma 3.3 implies the existence of $y_{0}, y_{1} \in H^{0}(\widetilde{\omega})$ such that $v_{\widetilde{P}}\left(y_{0}\right)=0$ and $v_{\widetilde{P}}\left(y_{1}\right)=1$ or 2 . But $H^{0}(\widetilde{\omega}) \subset H^{0}(\omega)$ and $v_{\widetilde{P}}=v_{P}$ as functions by the very definition. So there are $y_{0}, y_{1} \in H^{0}(\omega)$ such that $v_{P}\left(y_{0}\right)=0$ and $v_{P}\left(y_{1}\right)=1$ or 2. Lemma 3.2(iii) implies that $W_{x}^{n} \rightarrow V_{x, P}^{n} / \mathcal{C}_{P}^{n}$ is surjective for $n \geq 1$.

Lemma 3.6. Let $P \in C$ be a unibranch point of multiplicity at least 3 . Let $\widetilde{C}$ be the curve obtained from $C$ by resolving $P$ and let $\widetilde{g}$ be its genus. Let $\varphi: \widetilde{C} \rightarrow C$ be the natural morphism and $\widetilde{\omega}:=\varphi_{*}\left(\omega_{\widetilde{C}}\right)$. If $\widetilde{C}$ is hyperelliptic and $\widetilde{g} \geq 2$, then

$$
\operatorname{Sym}^{n} H^{0}(\omega) \rightarrow H^{0}\left(\widetilde{\omega}^{n}\right)
$$

is surjective for every $n \geq 1$.

Proof. Since $\widetilde{C}$ is hyperelliptic and $\widetilde{g} \geq 2$, there is $a \in k(C)$ such that $\operatorname{deg}\left(\operatorname{div}_{0}(a)\right)=$ 2 and

$$
H^{0}(\widetilde{\omega})=\left\langle y, a y, \ldots, a^{\widetilde{g}-1} y\right\rangle
$$

for a certain $y \in H^{0}(\widetilde{\omega})$. First, recall definition (15) for what we mean by $H^{0}(\widetilde{\omega})^{n}$. On the one hand, $\operatorname{dim}\left(H^{0}(\widetilde{\omega})^{n}\right)=n(\widetilde{g}-1)+1$ due to (12). On the other hand, if $n \geq 2$, then $\operatorname{dim}\left(H^{0}\left(\widetilde{\omega}^{n}\right)\right)=h^{0}\left(\widetilde{\omega}^{n}\right)=(2 n-1)(\widetilde{g}-1)$ owing to Riemann-Roch and Lemma 3.4. So, if $n \geq 2$, there are elements in $H^{0}\left(\widetilde{\omega}^{n}\right)$ which are not in $H^{0}(\widetilde{\omega})^{n}$. We will show that these elements are in $H^{0}(\omega)^{n}$ by proving that $\widetilde{W}_{y, n} \subset W_{y}^{n}$.

Let $\widetilde{P} \in \widetilde{C}$ be the point which lies over $P$ and let $\psi:=(1: a): \widetilde{C} \rightarrow \mathbb{P}^{1}$ be the degree 2 morphism. We will consider two cases.

1. $\widetilde{P}$ is a ramification point of $\psi$.

Let $\bar{P} \in \bar{C}$ be the point which lies over $\widetilde{P}$ and $P$. In this case we may take

$$
\operatorname{div}(a)=2 \bar{P}-\bar{Q}-\bar{R},
$$

where $\bar{Q}, \bar{R}$ lie over nonsingular points, say $Q, R$, of $C$.

Since the multiplicity of $P$ is at least 3 , one is able to consider the partial normalization map $\phi: C^{*} \rightarrow C$, where $C^{*}$ is obtained from $C$ by replacing $P$ by a point $P^{*}$ of multiplicity 3 for which the maximal ideal agrees with the conductor. In other words, if $\mathcal{O}^{*}:=\phi_{*}\left(\mathcal{O}_{C^{*}}\right)$, then $\mathcal{O}_{P}^{*}=t_{P}^{3} \overline{\mathcal{O}}_{P}$. Set $\omega^{*}:=\phi_{*}\left(\omega_{C^{*}}\right)$. If $h^{1}\left(\mathcal{O}^{*}(Q+R)\right)=h^{1}\left(\mathcal{O}^{*}(Q)\right)$, then $h^{0}\left(\mathcal{O}^{*}(Q+R)\right)>h^{0}\left(\mathcal{O}^{*}(Q)\right) \geq h^{0}\left(\mathcal{O}^{*}\right)=1$ because $\operatorname{deg}\left(\mathcal{O}^{*}(Q+R)\right)=\operatorname{deg}\left(\mathcal{O}^{*}(Q)\right)+1$. Now if $Q^{*}$ and $R^{*}$ are the points of $C^{*}$ lying over $Q$ and $R$, then $\operatorname{deg}_{C^{*}}\left(\mathcal{O}_{C^{*}}\left(Q^{*}+R^{*}\right)\right)=2$ and $h^{0}\left(\mathcal{O}_{C^{*}}\left(Q^{*}+R^{*}\right)\right)=$ $h^{0}\left(\mathcal{O}^{*}(Q+R)\right) \geq 2$. It follows that $C^{*}$ is hyperelliptic, which cannot happen 
because it has a point $P^{*}$ with multiplicity 3 (actually $P^{*}$ is even non-Gorenstein) but hyperelliptic curves have double points at worst [5, Prop. 2.6.(2)].

Therefore $h^{1}\left(\mathcal{O}^{*}(Q+R)\right)<h^{1}\left(\mathcal{O}^{*}(Q)\right)$, that is, $H^{0}\left(\omega^{*}(-Q-R)\right) \varsubsetneqq H^{0}\left(\omega^{*}(-Q)\right)$. By construction $\omega_{Q}^{*}=\widetilde{\omega}_{Q}$ and $\omega_{R}^{*}=\widetilde{\omega}_{R}$. Besides, (12) and (13) imply $\widetilde{\omega}_{Q}=$ $\mathcal{O}_{Q} a^{\widetilde{g}-1} y$ and $\widetilde{\omega}_{R}=\mathcal{O}_{R} a^{\widetilde{g}-1} y$. Then there exists $b \in k(C)$ such that $b y \in H^{0}\left(\omega^{*}\right)$ and with $v_{Q}(b)=-\widetilde{g}+2$ and $v_{R}(b)=-\widetilde{g}+1$.

Now set

$$
B:=\left\{1, a, \ldots, a^{n(\widetilde{g}-1)}, b a^{2}, \ldots, b a^{(n-1)(\widetilde{g}-1)}\right\} .
$$

We claim $\widetilde{W}_{y, n}=\langle B\rangle \subset W_{y}^{n}$. In fact, first, $B$ is a $k$-linearly independent set, as one can easily see by computing $v_{Q}$ and $v_{R}$ of its elements. Second, $|B|=$ $(2 n-1)(\hat{g}-1)=h^{0}\left(\widetilde{\omega}^{n}\right)=\operatorname{dim}\left(\widetilde{W}_{y, n}\right)$. Third, $B \subset \widetilde{W}_{y, n}$. Indeed, the $a^{i}$, s are clearly in $W_{y, n}$ and the $b a^{i}$ 's are in $\widetilde{V}_{y, S}^{n}$ for $n \geq 2$ and $S \neq P$ because $\widetilde{\omega}$ and $\omega^{*}$ agree outside $P$, by $\in H^{0}\left(\omega^{*}\right)$ and so $b \in \widetilde{V}_{y, S}$ for $S \neq P$. Let us show that the $b a^{i}$ s are in $\widetilde{V}_{y, P}$. From (12) and (13) we have that $\widetilde{\omega}_{P}=\overline{\mathcal{O}}_{P} y$, in particular that $v_{P}(y)=0$ as seen in the proof of Lemma 3.3. Now by $\in H^{0}\left(\omega^{*}\right)$, and so, according to (8), its pole at $P$ has order at most $\alpha_{P^{*}}=3$. Hence $v_{P}(b)=v_{P}(b y) \geq-3$. Therefore $v_{P}\left(b a^{2}\right)=v_{P}\left(a^{2}\right)+v_{P}(b) \geq 4-3>0$. So $b a^{2} \in \overline{\mathcal{O}}_{P}=\widetilde{V}_{y, P}$. This implies that all the $b a^{i}$, s are in $\widetilde{V}_{y, P}$. So we have proved $\widetilde{W}_{y, n}=\langle B\rangle$. Since $H^{0}\left(\omega^{*}\right) \subset H^{0}(\omega)$ it follows that $b \in W_{y}$, and since $H^{0}(\widetilde{\omega}) \subset H^{0}(\omega)$ it follows that the $a^{i}$ 's are in $W_{y}$. So $B \subset W_{y}^{n}$ as desired, and we are done with the first case.

2. $\widetilde{P}$ is not a ramification point of $\psi$.

Now we consider $C^{*}$, the curve obtained from $C$ by replacing $P$ by a point $P^{*}$ of multiplicity 2 for which the maximal ideal agrees with the conductor. We claim $C^{*}$ is nonhyperlliptic. Indeed, otherwise we would be able to take $a \in \mathcal{O}_{C^{*}, P^{*}}$. But if so, subtracting $a$ by a suitable constant we may suppose it is not a unit in $\mathcal{O}_{C^{*}, P^{*}}$ and so $v_{P}(a)=2$, but this contradicts the fact that $\widetilde{P}$ is not a ramification point of $\psi$. Actually, it is easy to see that $\left|\mathcal{O}_{C^{*}}\langle 1, a\rangle\right|$ is a $g_{3}^{1}$ on $C^{*}$ with a nonremovable base point $P^{*}$ and so $C^{*}$ is trigonal.

Then we proceed verbatim as in Case 1 up to the following change: we take $\operatorname{div}(a)=\bar{P}+\bar{P}_{1}-\bar{Q}-\bar{R}$ with $\bar{P}_{1} \neq \bar{P}$ and so $v_{P}(a)=1$; we have $\alpha_{P^{*}}=2$ and hence $v_{P}(b)=v_{P}(b y) \geq-2$. Therefore $v_{P}\left(b a^{2}\right)=v_{P}\left(a^{2}\right)+v_{P}(b) \geq 2-2=0$.

Theorem 3.7. Let $C$ be a nonhyperelliptic curve whose non-Gorenstein points are unibranch. Then the homomorphisms

$$
\operatorname{Sym}^{n} H^{0}(C, \omega) \longrightarrow H^{0}\left(C, \omega^{n}\right)
$$

are surjective.

Proof. We proceed by induction on the number of non-Gorenstein points of $C$. Assume first that there is only one non-Gorenstein point $P \in C$. Let $\widetilde{C}$ be the curve obtained from $C$ by resolving $P$ and let $\widetilde{g}$ be its genus. Let $\varphi: \widetilde{C} \rightarrow C$ be the natural morphism and $\widetilde{\omega}:=\varphi_{*}\left(\omega_{\widetilde{C}}\right)$.

If $\widetilde{g}=0$, then $h^{0}\left(\widetilde{\omega}^{n}\right)=0$ for $n \geq 1$ and $h^{1}\left(\omega^{n}\right)=0$ for $n \geq 2$ due to Lemma 3.4 because $g \geq 2$ since $C$ is non-Gorenstein. Besides, from Lemma 3.2(i) we have 
$\operatorname{dim}\left(W_{x}^{n}\right) \geq \operatorname{dim}\left(V_{x, P}^{n} / t_{P}^{-(2 n-1)} \mathcal{C}_{P}^{n}\right)$ for $n \geq 2$, and we have already seen that $\widetilde{V}_{x, P}=$ $\mathcal{C}_{P}$. Assuming $n \geq 2$, these statements lead us to

$$
\begin{aligned}
\operatorname{dim}\left(H^{0}\left(\omega^{n}\right)\right) & =h^{0}\left(\omega^{n}\right)=h^{0}\left(\omega^{n}\right)-h^{0}\left(\widetilde{\omega}^{n}\right) \\
& =\operatorname{deg}\left(\omega^{n}\right)-\operatorname{deg}\left(\widetilde{\omega}^{n}\right)-h^{1}\left(\widetilde{\omega}^{n}\right) \\
& =\operatorname{dim}\left(\omega_{P}^{n} / \widetilde{\omega}_{P}^{n}\right)-(2 n-1) \\
& =\operatorname{dim}\left(V_{x, P}^{n} / \widetilde{V}_{x, P}^{n}\right)-(2 n-1) \\
& =\operatorname{dim}\left(V_{x, P}^{n} / \mathcal{C}_{P}^{n}\right)-(2 n-1) \\
& =\operatorname{dim}\left(V_{x, P}^{n} / t_{P}^{-(2 n-1)} \mathcal{C}_{P}^{n}\right) \\
& \leq \operatorname{dim}\left(W_{x}^{n}\right)=\operatorname{dim}\left(H^{0}(\omega)^{n}\right)
\end{aligned}
$$

Now, recalling the definition of $H^{0}(\omega)^{n}$ in (5), we have the natural sequence of morphisms

$$
\operatorname{Sym}^{n} H^{0}(\omega) \stackrel{\phi}{\longrightarrow} H^{0}(\omega)^{n} \stackrel{i}{\longrightarrow} H^{0}\left(\omega^{n}\right)
$$

where $\phi$ is surjective and $i$ is the inclusion and hence injective. Now the theorem trivially holds for $n=1$, and we have just seen that $\operatorname{dim}\left(H^{0}\left(\omega^{n}\right)\right) \leq \operatorname{dim}\left(H^{0}(\omega)^{n}\right)$ if $n \geq 2$, so $H^{0}\left(\omega^{n}\right)$ and $H^{0}(\omega)^{n}$ have to agree if $n \geq 2$, that is, $\operatorname{Sym}^{n} H^{0}(\omega)$ surjects $H^{0}\left(\omega^{n}\right)$ if $n \geq 2$, and hence the theorem holds if $C$ has just one non-Gorenstein point and $\widetilde{g}=0$.

If $\widetilde{g}=1$, then $\omega_{\widetilde{C}} \cong \mathcal{O}_{\widetilde{C}}$. In particular, $H^{0}(\widetilde{\omega})^{n}=H^{0}\left(\widetilde{\omega}^{n}\right)$. Besides, if $\widetilde{g}=1$, then there exists $y_{0} \in H^{0}(\widetilde{\omega}) \subset H^{0}(\omega)$ such that $v_{P}\left(y_{0}\right)=0$ owing to Lemma 3.3(i). Hence Lemma 3.2(ii) implies that $W_{x}^{n} \rightarrow V x, P^{n} / t^{-1} \mathcal{C}_{P}^{n}$ is surjective. Since $\widetilde{V}_{x, P}=\mathcal{C}_{P}$ and $\widetilde{W}_{x}=W_{x} \cap \widetilde{V}_{x, P}$ it follows that $W_{x}^{n} / \widetilde{W}_{x}^{n} \rightarrow V x, P^{n} / t_{P}^{-1} \mathcal{C}_{P}^{n}$ remains surjective. These statements lead us to

$$
\begin{aligned}
\operatorname{dim}\left(H^{0}\left(\omega^{n}\right) / H^{0}(\widetilde{\omega})^{n}\right) & =\operatorname{dim}\left(H^{0}\left(\omega^{n}\right) / H^{0}\left(\widetilde{\omega}^{n}\right)\right) \\
& =h^{0}\left(\omega^{n}\right)-h^{0}\left(\widetilde{\omega}^{n}\right) \\
& =\operatorname{deg}\left(\omega^{n}\right)-\operatorname{deg}\left(\widetilde{\omega}^{n}\right)-h^{1}\left(\widetilde{\omega}^{n}\right) \\
& =\operatorname{dim}\left(\omega_{P}^{n} / \widetilde{\omega}_{P}^{n}\right)-1 \\
& =\operatorname{dim}\left(V_{x, P}^{n} / \widetilde{V}_{x, P}^{n}\right)-1 \\
& =\operatorname{dim}\left(V_{x, P}^{n} / \mathcal{C}_{P}^{n}\right)-1 \\
& =\operatorname{dim}\left(V_{x, P}^{n} / t_{P}^{-1} \mathcal{C}_{P}^{n}\right) \\
& \leq \operatorname{dim}\left(W_{x}^{n} / \widetilde{W}_{x}^{n}\right) \\
& =\operatorname{dim}\left(H^{0}(\omega)^{n} / H^{0}(\widetilde{\omega})^{n}\right)
\end{aligned}
$$

Therefore $\operatorname{dim}\left(H^{0}\left(\omega^{n}\right)\right) \leq \operatorname{dim}\left(H^{0}(\omega)^{n}\right)$, and by the same argument used for $\widetilde{g}=0$, we conclude that the theorem also holds if $C$ has just one non-Gorenstein point and $\widetilde{g}=1$.

Now $P$ has multiplicity at least 3 for it is non-Gorenstein, and $\widetilde{C}$ is Gorenstein because $P$ is the only non-Gorenstein point of $C$. Thus if $\widetilde{g} \geq 2$, either $\widetilde{C}$ is hyperelliptic and so $\operatorname{Sym}^{n} H^{0}(\omega) \rightarrow H^{0}\left(\widetilde{\omega}^{n}\right)$ is surjective owing to Lemma 3.6 or else $\widetilde{C}$ is nonhyperelliptic Gorenstein and so $\operatorname{Sym}^{n} H^{0}(\widetilde{\omega}) \rightarrow H^{0}\left(\widetilde{\omega}^{n}\right)$ is surjective owing to Theorem 2.7. Besides, $\operatorname{Sym}^{n} H^{0}(\omega) \rightarrow H^{0}\left(\omega^{n}\right) / H^{0}\left(\widetilde{\omega}^{n}\right)$ is surjective due to Lemma 3.5. Therefore the theorem holds if $C$ has just one non-Gorenstein point. 
If $C$ has many non-Gorenstein points, define $\widetilde{C}$ by resolving just one of them. Then $\operatorname{Sym}^{n} H^{0}(\widetilde{\omega}) \rightarrow H^{0}\left(\widetilde{\omega}^{n}\right)$ by induction and $\operatorname{Sym}^{n} H^{0}(\omega) \rightarrow H^{0}\left(\omega^{n}\right) / H^{0}\left(\widetilde{\omega}^{n}\right)$ owing to Lemma 3.5. We are done.

Remark 3.8. Though it was not our aim, one can combine Theorem 2.7 with Theorem 3.7 to get the following result: if $C$ is a nonhyperelliptic curve with at most one multibranch non-Gorenstein point which is also almost Gorenstein, then $\operatorname{Sym}^{n} H^{0}(\omega) \rightarrow H^{0}\left(\omega^{n}\right)$ is surjective for $n \geq 1$. The proof is basically the same as Theorem 3.7, just using Theorem 2.7 in its full generality.

\section{ACKNOWLEDGMENTS}

This article continues [5], a joint work with Steven L. Kleiman, whom we thank very much for an invitation to MIT and for regular email discussions, after which many suggestions were built into this paper. We also thank the referee for many corrections, suggestions and observations. The author is partially supported by CNPq grant numbers PDE 200999/2005-2 and PQ 304919/2009-8.

\section{REFERENCES}

[1] Arbarello, E., Cornalba, M., Griffiths, P. A., and Harris, J., "Geometry of Algebraic Curves," Springer-Verlag, 1985. MR770932 (86h:14019)

[2] Barucci, V., and Fröberg, R., One-Dimensional Almost Gorenstein Rings, J. Alg. 188 (1997), 418-442. MR.1435367 (98a:13033)

[3] Barucci, V., D'Anna, M., and Fröberg, R., Analytically Unramified One-Dimensional Semilocal Rings and Their Value Semigroups, J. Pure Appl. Alg. 147 (2000), 215-254. MR:1747441 (2001g:13054)

[4] Eisenbud, D., Koh, J., and Stillman, M. (appendix with Harris, J.), Determinantal Equations for Curves of High Degree, Amer. J. Math. 110 (1988), 513-539. MR944326 (89g:14023)

[5] Kleiman, S. L., and Martins, R. V., The Canonical Model of a Singular Curve, Geom. Dedicata 139 (2009), 139-166. MR2481842(2010d:14038)

[6] Matsuoka, T., On the Degree of Singularity of One-Dimensional Analytically Irreducible Noetherian Local Rings, J. Math. Kyoto Univ. 11-3 (1971), 485-494. MR0285526 $(44: 2744)$

[7] Rosenlicht, M., Equivalence Relations on Algebraic Curves, Annals of Math. (2) 56 (1952), 169-191. MR0048856 (14:80c)

[8] Rosa, R., Stöhr, K.-O., Trigonal Gorenstein Curves, J. Pure Appl. Alg. 174 (2002), 187-205. MR 1921820 (2003k:14037)

[9] Stöhr, K.-O., On the Poles of Regular Differentials of Singular Curves, Bull. Brazilian Math. Soc. 24 (1993), 105-135. MR1224302 (94e:14035)

[10] Stöhr, K.-O., Hyperelliptic Gorenstein Curves, J. Pure Appl. Alg. 135 (1999), 93-105. MR:1667447 (99m:14049)

Departamento de Matemática, Instituto de Ciencias Exatas, Universidade Federal de Minas Gerais, Av. Antônio Carlos 6627, 30123-970 Belo Horizonte MG, Brazil

E-mail address: renato@mat.ufmg.br 